\title{
Hypernatremia increases mortality in patients admitted by COVID-19: A Retrospective longitudinal study with 1000 patients
}

Jonas Wolf ( $\square$ jonasmwolf@gmail.com )

Lutheran University: Universidade Luterana do Brasil https://orcid.org/0000-0001-7577-464X

Francine Possebon Berlesi

Moinhos de Vento Hospital: Hospital Moinhos de Vento

Tiane Crestani dos Santos

Moinhos de Vento Hospital: Hospital Moinhos de Vento

Andressa Barreto Glaeser

Moinhos de Vento Hospital: Hospital Moinhos de Vento

Vagner Ricardo Lunge

ULBRA: Universidade Luterana do Brasil

Juçara Gasparetto Maccari

Hospital Moinhos de Vento

Luiz Antonio Nasi

Moinhos de Vento Hospital: Hospital Moinhos de Vento

\section{Research Article}

Keywords: COVID-19, Dysnatremia, Hypernatremia, Death

Posted Date: February 17th, 2022

DOI: https://doi.org/10.21203/rs.3.rs-1361819/v1

License: (9) (i) This work is licensed under a Creative Commons Attribution 4.0 International License.

Read Full License 


\section{Abstract}

Coronavirus disease 2019 (COVID-19) pandemic spread rapidly and this scenario is concerning worldwide. In hospitalized patients, dysnatremia (hyponatremia and/or hypernatremia) is the most common electrolyte disturbance, being reported in $30-40 \%$ of cases and associated with a poor prognosis. This retrospective longitudinal study aimed to evaluate the association of dysnatremia with mortality in hospitalized patients infected with COVID-19. We analyzed data from hospital records of 1000 patients with COVID-19 (median age, 62.5 years; $57.1 \%$ men), including 109 (10.9\%) deaths. KaplanMeier survival curves and Cox proportional hazard models with Hazard Ratio (HR) with $95 \%$ confidence intervals $(95 \% \mathrm{Cl})$ were applied to confirm the association between dysnatremia (hyponatremia and/or hypernatremia) and death. Hypernatremia was detected in 83 (76.1\%) of the patients who died, with a cumulative reduction in survival $(p<0.01)$ and an increased risk for death by 2.4 fold $(95 \% \mathrm{Cl} 1.4-2.9)$. In the multivariable analysis, hypernatremia was the main factor associated with increased mortality (HR:1.50; 95\%Cl:1.23-1.81). Other factors also associated with death were long length of stay (LOS) (HR:1.54; 95\%Cl:1.21-1.78), old age (HR:1.63; 95\%Cl:1.28-1.88), and kidney disease (HR:1.77; $95 \% \mathrm{Cl}: 1.21-3.30)$. Hypernatremia during hospitalization is an important risk factor for poor prognosis and an increased risk (2.4) of mortality. LOS, old age, and kidney disease could also be used for risk stratification in patients with COVID-19.

\section{Introduction}

Severe acute respiratory syndrome - coronavirus 2 (SARS-CoV-2) is a very transmissible microorganism with a high rate of dissemination in the last two years [1]. SARS-CoV2 current worldwide pandemic has already affected more than 295 million people with more than 5 million deaths. In Brazil, more than 22 million people were infected and presented COVID-19, generating a large number of hospital admissions and more than 600,000 deaths [2].

COVID-19 is a systemic disease with a predominance of respiratory symptoms. Since it is a new disease, other short-term and long-term effects have been studied in the current pandemic [3]. A previous study evaluated 24,410 COVID-19 patients and the main symptoms were fever (78\%), cough (56\%), and fatigue (31\%), with $19 \%$ of all hospitalized patients requiring non-invasive ventilation and $9 \%$ requiring invasive ventilation [4]. Also, a high incidence of pulmonary edema and embolism has been observed as opposed to similar (viral) respiratory tract infections [5,6]. COVID-19 hospitalized patients seem to present extrapulmonary manifestations too, such as water and electrolytes disorders [7-9].

Dysnatremia (serum sodium level $>145 \mathrm{mmol} / \mathrm{L}$ or $<135 \mathrm{mmol} / \mathrm{L}$ ) is the most common electrolyte disturbance that has been associated with a worse prognosis for hospitalized COVID-19 patients [10-15]. It was also previously demonstrated that hospitalized patients with community-acquired pneumonia (CAP) caused by other pathogenic microorganisms presenting hyponatremia and hypovolemia have a higher risk of death [13]. Hyponatremia is also a disturbance with an incidence rate of up to $30 \%$ in hospitalized patients presenting poor prognosis in pneumonia outcome [16-20]. More recently, 
hypernatremia during the hospitalization period was associated with a worse prognosis in COVID-19 patients $[7,8,13]$. The present study aimed to evaluate the association of dysnatremia with a high risk of death in COVID-19 hospitalized patients.

\section{Methods}

\section{Data Collection}

This was a retrospective longitudinal study carried out in Brazil, in the city of Porto Alegre, at the Hospital Moinhos de Vento. All participants signed an informed consent form and this study was approved from Institutional Review Boards of National Health Council of Brazil (approval number 4.497.118). The study analyzed data from hospital records of 1000 patients over 18 years of age, regardless of gender, ethnicity, previous comorbidities, and length of hospital stay hospitalized with COVID-19, confirmed by RT-PCR, between March and December 2020. All patients were routinely evaluated for plasma sodium concentration and the number of the dosages varied according to the clinical status and the length of stay (LOS). Patients with at least three sodium analyses were further selected to compare different dosages, including the first one (performed in the hospital admission) and the two presenting the highest and the lowest values. Sodium values were used to define the clinical category of the patient: hyponatremia (<135 mmol/L), eunatremia (135-145 mmol/L), and hypernatremia (> $140 \mathrm{mmol} / \mathrm{L})$ $[18,20]$.

\section{Statistical Analysis}

Kolmogorov-Smirnov with Lilliefors correction and Shapiro-Wilk tests were performed to evaluate the normality of the continuous data. All continuous data are presented as median followed by the interquartile range (IQRs) and differences between groups were tested with the Mann-Whitney $\mathrm{U}$ and Kruskal-Wallis (with Tukey posthoc) tests. Categorical variables are reported as numbers (percentage), and a chi-square test was used for study comparisons. Kaplan-Meier survival curves were produced for serum sodium, length of stay (LOS), and mortality with log-rank Mantel-Cox chi-square distribution. Cox proportional hazard models with covariates were used to assess the association of sodium concentrations with mortality. A priori confounders considered in the multivariable analysis were LOS, sodium measures (admission, lower, and higher), sex, age, and comorbidities (heart disease, hypertension, pulmonary, diabetes, kidney, cerebrovascular disease, central nervous system disease, cancer, and arthritis). Hazard ratio (HR) and $95 \%$ confidence intervals $(95 \% \mathrm{Cl})$ were estimated and significance was accepted at $p$-values $<0.05$ for all tests. SPSS, Version 23.0 for Windows (SPSS Inc., Chicago, IL, USA), and R software (R Foundation for Statistical Computing, Vienna, Austria; $<$ http://www.R-project.org>) were used for data analysis.

\section{Results}

\section{Baseline Characteristics of COVID-19 patients}


This study included 1000 patients, 571 male (57.1\%) and 429 female (42.9\%), with a median age of 62.5 years (IQR: 47.0-75.0), and LOS of 8.0 days (IQR: 5.0-14.0). Of these patients, 335 (33.5\%) were admitted to the ICU and 109 died (52.3\% men and $47.7 \%$ women; $p=0.28)$. Age and LOS were significantly higher in patients who died $(p<0.01)$. The most common comorbidities were hypertension $(46.2 \%)$, diabetes $(19.1 \%)$, pulmonary disease $(17.8 \%)$, heart disease $(17.6 \%)$, depression $(8.7 \%)$, cancer (8.6\%), kidney disease (7.0\%), central nervous system disease (5.6\%), cerebrovascular disease $(5.0 \%)$, arthritis (1.6\%), and liver disease (1.1\%). Hypertension, diabetes, pulmonary disease, heart disease, cancer, kidney disease, central nervous system disease, and cerebrovascular disease were significantly more frequent in patients who died than in those who survived (Table 1). 
Table 1

Bivariate analysis of the characteristics between survived and dead patients with COVID-19 included in this study

\begin{tabular}{|c|c|c|c|c|c|c|c|}
\hline \multirow[t]{2}{*}{ Variables } & \multicolumn{2}{|c|}{ Total $(n=1000)$} & \multicolumn{2}{|c|}{ Survived $(n=891)$} & \multicolumn{2}{|c|}{ Dead $(n=109)$} & \multirow[t]{2}{*}{$p$-values } \\
\hline & $\mathbf{n}$ & $\%$ & $\mathbf{n}$ & $\%$ & $\mathbf{n}$ & $\%$ & \\
\hline \multicolumn{8}{|l|}{ Sex } \\
\hline Female & 429 & 42.9 & 377 & 42.3 & 52 & 47.7 & 0.28 \\
\hline Male & 571 & 57.1 & 514 & 57.7 & 57 & 52.3 & \\
\hline Median age (IQR) & \multicolumn{2}{|c|}{$62.5(47.0-75.0)$} & \multicolumn{2}{|c|}{$59.0(45.0-71.0)$} & \multicolumn{2}{|c|}{$85.0(80.0-91.0)$} & $<0.01$ \\
\hline Median length of stay (IQR) & \multicolumn{2}{|c|}{$8.0(5.0-14.0)$} & \multicolumn{2}{|c|}{$8.0(5.0-12.0)$} & \multicolumn{2}{|c|}{$18.0(10.0-36.0)$} & $<0.01$ \\
\hline \multicolumn{8}{|l|}{ Comorbidities } \\
\hline Heart disease & 176 & 17.6 & 127 & 14.3 & 49 & 45.0 & $<0.01$ \\
\hline Hypertension & 462 & 46.2 & 390 & 43.8 & 72 & 66.1 & $<0.01$ \\
\hline Pulmonary disease & 178 & 17.8 & 151 & 16.9 & 27 & 24.8 & 0.04 \\
\hline Diabetes & 191 & 19.1 & 157 & 17.6 & 34 & 31.2 & $<0.01$ \\
\hline Chronic Kidney disease & 70 & 7.0 & 42 & 4.7 & 28 & 25.7 & $<0.01$ \\
\hline Liver disease & 11 & 1.1 & 9 & 1.0 & 2 & 1.8 & 0.43 \\
\hline Cerebrovascular disease & 50 & 5.0 & 29 & 3.3 & 21 & 19.3 & $<0.01$ \\
\hline CNS disease & 56 & 5.6 & 30 & 3.4 & 26 & 23.9 & $<0.01$ \\
\hline Cancer & 86 & 8.6 & 67 & 7.5 & 19 & 17.4 & $<0.01$ \\
\hline Depression & 87 & 8.7 & 80 & 9.0 & 7 & 6.4 & 0.37 \\
\hline Arthritis & 16 & 1.6 & 12 & 1.3 & 4 & 3.7 & 0.06 \\
\hline \multicolumn{8}{|l|}{ IQR: Interquartile range. } \\
\hline & & & & & & & \\
\hline
\end{tabular}

\section{Association between dysnatremia and death}

Hypernatremia was significantly more frequent in deaths than in survivors in the three measurements (6.7\% vs $1.0 \%$ on admission, $3.7 \%$ vs $0.0 \%$ at the lowest concentration, and $76.1 \%$ vs $9.7 \%$ at the highest concentration, $p<0.01$ ) (Table S1). In the quantitative analysis, sodium concentrations were statistically higher in patients who died than in survivors at admission (median: 138.0, IQR: 135.0-145.0 vs median: 137.0; IQR: 135.0-139.0, $p=0.04$, Table S2) and at the highest concentration (median: 148.0, IQR: 146.0- 
153.0 vs median: 140.0; IQR: 138.0-142.0, $p<0.01$, Fig. 1, Table S2), respectively. However, there was no significant difference between low sodium concentrations among patients who died compared with those who survived (median: 135.0, IQR: 132.0-137.0 vs median: 135.0, IQR: 133.0-137.0; $p=0.80$ ) (Table S2).

\section{Length of stay (LOS) and sodium concentration}

LOS was evaluated concerning sodium concentrations. On admission, the LOS had medians of 10.0 (IQR: 6.0-16.0) for hyponatremia, 7.0 (IQR: 5.0-13.0) for eunatremia, and 13.0 (IQR: 7.5-35.0) for hypernatremia (Table S3). At the lowest sodium concentration, the LOS was 11.0 (IQR: 7.0-21.0) for hyponatremia, 7.0 (IQR: 4.0-10.0) for eunatremia, and 7.5 (IQR: 2.5-10.5) hypernatremia (Table S3). At the highest sodium concentration, the LOS was 7.0 (IQR: 5.0-10.0) for hyponatremia, 7.0 (IQR: 5.0-11.0) for eunatremia, and 29.0 (IQR: 16.0-48.0) for hypernatremia (Fig. 2, Table S3). Patients with hypernatremia had higher LOS at admission and the highest sodium concentration $(p<0.01)$ (Fig. 2$)$. However, patients with hyponatremia had significantly more LOS in the evaluation of lower sodium concentrations (Table S3).

\section{Changes in sodium concentration, LOS, and risk of death}

In the Kaplan Meier analysis, the three sodium concentrations were evaluated (admission, lower and higher concentrations) concerning the LOS. On admission, hypernatremia was present in 7 deaths (6.7\%), of which showed a cumulative reduction in survival that lasted 72 days $(p=0.05)$. In the analysis with the lower sodium concentrations, hypernatremia was present in 4 deaths (3.7\%), of which they presented a cumulative reduction in survival that lasted only 10 days $(p<0.01)$. In the analysis with higher sodium concentrations, hypernatremia was present in 83 deaths $(76.1 \%)$, of which presented a cumulative reduction in survival that lasted 127 days $(p<0.01$ ) (Table S4), being the most robust analysis, indicating that hypernatremia significantly reduces survival and significantly increased the cumulative risk by 2.4 fold (95\%Cl: 1.4-2.9, $p<0.01)$ (Fig. 3).

\section{Hypernatremia, the longer length of stay, older age, and kidney disease are associated independently with COVID-19 mortality}

In the multivariate analysis by Cox regression, the variables independently associated with mortality were: Hypernatremia (HR: 1.50; 95\%Cl: 1.23-1.81; $p<0.01$ ), longer LOS (HR: 1.54; 95\%Cl: $1.21-1.78 ; p<0.01$ ), older age (HR: $1.63 ; 95 \% \mathrm{Cl}: 1.28-1.88 ; p<0.01)$ and kidney disease (HR: $1.77 ; 95 \% \mathrm{Cl}: 1.21-3.30 ; p<0.01)$ (Table 2). These factors show great potential as predictors of mortality, increasing the probability of death by $50 \%$ for hypernatremia, $54 \%$ for longer LOS, $63 \%$ for older age, and $77 \%$ for kidney disease. 
Table 2

Multivariable associations of risk factors with in-hospital mortality among COVID-19 patients

\begin{tabular}{|llllll|}
\hline Variables & $\begin{array}{l}\text { Wald Chi- } \\
\text { Square }\end{array}$ & $\begin{array}{l}\text { Hazard } \\
\text { Ratio }\end{array}$ & $\begin{array}{l}\text { lower } \\
\text { Cl95\% }\end{array}$ & $\begin{array}{l}\text { Higher } \\
\text { Cl95\% }\end{array}$ & $\begin{array}{c}\text { p- } \\
\text { values }\end{array}$ \\
\hline $\begin{array}{l}\text { Sodium concentration } \\
\text { (higher) }\end{array}$ & 21.61 & 1.50 & 1.23 & 1.81 & $<0.01$ \\
\hline $\begin{array}{l}\text { Sodium concentration } \\
\text { (admission) }\end{array}$ & 0.10 & 1.00 & 0.95 & 1.05 & 0.99 \\
\hline Sodium concentration (lower) & 0.01 & 1.00 & 0.94 & 1.06 & 0.92 \\
\hline Length of stay (LOS) & 15.29 & 1.54 & 1.21 & 1.78 & $<0.01$ \\
\hline Sex male & 0.40 & 0.87 & 0.56 & 1.34 & 0.53 \\
\hline Age (scale) & 60.78 & 1.63 & 1.28 & 1.88 & $<0.01$ \\
\hline Comorbidities & 1.84 & 1.38 & 0.87 & 2.18 & 0.17 \\
\hline Heart disease & 0.00 & 1.01 & 0.64 & 1.58 & 0.98 \\
\hline Hypertension & 1.40 & 1.33 & 0.83 & 2.14 & 0.24 \\
\hline Pulmonar & 0.44 & 1.17 & 0.74 & 1.84 & 0.51 \\
\hline Diabetes & $\mathbf{1 . 4 3}$ & 1.77 & 1.21 & 3.30 & $<0.01$ \\
\hline Kidney & 67.41 & 1.28 & 0.77 & 2.14 & 0.34 \\
\hline Cerebrovascular disease & 0.92 & 1.27 & 0.74 & 2.19 & 0.38 \\
\hline CNS & 0.76 & 1.09 & 0.63 & 1.87 & 0.77 \\
\hline Cancer & 0.21 & 1.15 & 0.40 & 3.35 & 0.79 \\
\hline Arthritis & 0.28 & & & & \\
\hline Significant $p$-values are highlighted in bold. & & & & & \\
\hline CNS: Central Nervous System & & & & & \\
\hline
\end{tabular}

\section{Discussion}

Dysnatremia is associated with increased mortality in patients with pneumonia caused by COVID-19 [8]. In the present study, hypernatremia was associated with a 50\% higher risk of mortality in multivariate analysis in patients admitted by COVID-19. We evaluated 1,000 patients hospitalized with COVID-19 and detected that hypernatremia was largely frequent, affecting $76.1 \%$ of individuals who died. Our results are consistent with those of other relevant investigations to date $[7,13-15,21]$.

The sodium levels disturbance is the most common electrolyte abnormality in clinical practice. Clinical data reported that hypernatremia is present in approximately $2.0 \%$ of patients older than 65 years but in 
almost $4.8 \%$ of those older than 75 years admitted to the hospital [22]. In a retrospective study involving 8,441 older patients admitted to the ICU, approximately $3.6 \%$ had hypernatremia on admission, and $15.3 \%$ developed hypernatremia during hospitalization; the mortality rate in those admitted with hypernatremia was approximately $33.3 \%$, while patients without hypernatremia had a mortality rate of $18.1 \%$ [23].

In a previous study that evaluated 111,545 sodium measures, hypernatremic and hyponatremic patients showed higher LOS than eunatremic. The mortality rates of hyponatremic and hypernatremic inpatients were $19 \%$ and $48 \%$, respectively. Disease severity as measured by LOS and mortality indicated these critical limits should not be broadened [24]. Hypernatremia has detrimental effects on various physiologic functions and was shown to be an independent risk factor for increased mortality in COVID-19 patients [25]. These results are in agreement with our findings, where hypernatremia and older age were associated with mortality by COVID-19.

Additionally, longer LOS, older age, and kidney disease were significant predictors for death. The association between hypernatremia, kidney disease, and higher LOS with mortality by COVID-19 was previously shown in a previous multicentric cohort that evaluated 4,664 patients [8]. These associations were observed in the present study, allowing a clinical characterization of COVID-19 patients in southern Brazil, reproducing the findings of risk factors for mortality. On the other hand, hyponatremia could indicate pulmonary involvement, whereas hypernatremia is associated with prolonged LOS and the need for intensive care/mechanical ventilation, particularly when resulting from prior hyponatremia [14].

In this COVID-19 cohort, hypernatremia and kidney disease were associated independently with mortality. Previous studies showed that is indirect and direct adverse consequences of high dietary sodium on the kidney. In patients with kidney disease, dietary sodium may have pivotal effects on hypertension control, proteinuria, immunosuppressant therapy, the efficacy of antiproteinuric pharmacologic therapy, and maintaining an optimal volume status. Dietary sodium intake is an important factor in patients with all stages of chronic kidney disease, counting those receiving dialysis therapy or those who have received a kidney transplant [25-28].

Many studies confirmed that both dysnatremia were independently associated with poor prognosis in patients hospitalized by COVID-19 [7,8,28,29]. Interestingly, in patients hospitalized with COVID-19, was detected that admittance hyponatremia was frequent; affecting more than $20 \%$ of patients, for a total of 957 subjects, and hypernatremia was found in only $3.7 \%$, a total of 174 subjects [8]. Another study, including 488 subjects with COVID-19, found an incidence of $24.6 \%$ of hyponatremia and $5.3 \%$ of hypernatremia [13]. Also, hypernatremia detected two days after admission and exposure to hypernatremia at any time point during hospitalization were associated with a 2.34 -fold and 3.05 -fold increased risk of death, respectively, compared to eunatremia. Hyponatremia at admission was linked with a 2.18-fold increase in the likelihood of needing ventilatory support [13]. These results showed the pivotal importance of sodium measures in patients hospitalized, including COVID-19 patients, mainly in ICU patients. 
This study has important limitations. This is a retrospective study and the consequential relationship between dysnatremia and mortality cannot be asserted. It is also not possible to assess the effect of hypernatremia treatment on the prognosis. Finally, the serum sodium was not corrected for glycemia. The main advantage of the study is a large number of patients were included, all of whom were admitted with RT-PCR-confirmed COVID-19. Also, all patients were followed-up throughout the entire period of hospitalization. In addition, all sodium measurements during follow-up were considered, computing the highest and lowest sodium values. In this sense, the stratification of patients on arrival at the hospital (considering clinical and laboratory tests, for example) makes it possible to identify those who are at greater risk of an unfavorable clinical outcome and makes it possible to improve monitoring and availability of resources for them $[1,28]$.

In conclusion, hypernatremia during hospitalization is an important risk factor for poor prognosis, being associated independently with a greater risk of mortality. Also, the other factors associated with COVID19 mortality were longer LOS, older age, and kidney disease. Serum sodium values, longer LOS, older age, and kidney disease could be used for risk stratification in patients with COVID-19. Physicians treating COVID-19 should be made aware that patients with dysnatremia are at a higher risk for death than those presenting with eunatremic. Intervention studies would be needed to ascertain whether correction of dysnatremia could improve clinical evolution.

\section{Declarations}

\section{Authorship}

Jonas M. Wolf, Franciele P. Berlesi, Tiane C. dos Santos, Andressa B. Glaeser, Juçara G. Maccari, and Luiz A. Nasi designed the study. Jonas M. Wolf performed the statistical analyses. Jonas M. Wolf, Franciele P. Berlesi, Tiane C. dos Santos, Andressa B. Glaeser, Vagner R. Lunge, Juçara G. Maccari, and Luiz A. Nasi wrote the first draft of the manuscript and contributed to the literature review and discussion of results. All authors contributed to and have approved the final manuscript.

\section{Funding}

This research did not receive any specific grant from funding agencies in the public, commercial, or notfor-profit sectors.

\section{Conflicts of interest}

The authors declare no conflicts of interest.

\section{References}

1. Donnelly JP, Wang XQ, Iwashyna TJ, Prescott HC (2021). Readmission and Death After Initial Hospital Discharge Among Patients With COVID-19 in a Large Multihospital System. JAMA. 
325(3):304-306. doi: 10.1001/jama.2020.21465.

2. John Hopkins University of Medicine. Coronavirus Resource Center. Available: <https://coronavirus.jhu.edu/map.html> Accessed February 08, 2022.

3. Zhou F, Yu T, Du R, Fan G, Liu Y, Liu Z, et al (2020). Clinical course and risk factors for mortality of adult inpatients with COVID-19 in Wuhan, China: a retrospective cohort study. Lancet. 395(10229):1054-1062. doi: 10.1016/S0140-6736(20)30566-3. Erratum in: Lancet. 2020;395(10229):1038.

4. Grant MC, Geoghegan L, Arbyn M, Mohammed Z, McGuinness L, Clarke EL, et al (2020). The prevalence of symptoms in 24,410 adults infected by the novel coronavirus (SARS-CoV-2; COVID-19): A systematic review and meta-analysis of 148 studies from 9 countries. PLoS One. 15(6):e0234765. doi: $10.1371 /$ journal.pone. 0234765 .

5. Bompard F, Monnier H, Saab I, Tordjman M, Abdoul H, Fournier L, et al (2020). Pulmonary embolism in patients with COVID-19 pneumonia. Eur Respir J. 56(1):2001365. doi: 10.1183/13993003.013652020.

6. de Maat S, de Mast Q, Danser AHJ, van de Veerdonk FL, Maas C (2020). Impaired Breakdown of Bradykinin and Its Metabolites as a Possible Cause for Pulmonary Edema in COVID-19 Infection. Semin Thromb Hemost. 46(7):835-837. doi: 10.1055/s-0040-1712960.

7. Atila C, Sailer CO, Bassetti S, Tschudin-Sutter S, Bingisser R, Siegemund M, et al (2021). Prevalence and outcome of dysnatremia in patients with COVID-19 compared to controls. Eur J Endocrinol. 184(3):409-418. doi: 10.1530/EJE-20-1374.

8. Ruiz-Sánchez JG, Núñez-Gil IJ, Cuesta M, Rubio MA, Maroun-Eid C, Arroyo-Espliguero R, et al (2020). Prognostic Impact of Hyponatremia and Hypernatremia in COVID-19 Pneumonia. A HOPECOVID-19 (Health Outcome Predictive Evaluation for COVID-19) Registry Analysis. Front Endocrinol (Lausanne). 11:599255. doi: 10.3389/fendo.2020.599255.

9. Hirsch JS, Uppal NN, Sharma P, Khanin Y, Shah HH, Malieckal DA, et al (2021). Prevalence and outcomes of hyponatremia and hypernatremia in patients hospitalized with COVID-19. Nephrol Dial Transplant. 36(6):1135-1138. doi: 10.1093/ndt/gfab067.

10. Lindner G, Funk GC, Schwarz C, Kneidinger N, Kaider A, Schneeweiss B, et al (2007). Hypernatremia in the critically ill is an independent risk factor for mortality. Am J Kidney Dis. 50(6):952-7. doi: 10.1053/j.ajkd.2007.08.016.

11. Lindner G, Funk GC (2013). Hypernatremia in critically ill patients. J Crit Care. 28(2):216.e11-20. doi: 10.1016/j.jcrc.2012.05.001.

12. Voets PJ, Frölke SC, Vogtländer NP, Kaasjager KA (2021). COVID-19 and dysnatremia: A comparison between COVID-19 and non-COVID-19 respiratory illness. SAGE Open Med. 9:20503121211027778. doi: $10.1177 / 20503121211027778$.

13. Tzoulis P, Grossman AB, Baldeweg SE, Bouloux P, Kaltsas G (2021). MANAGEMENT OF ENDOCRINE DISEASE: Dysnatraemia in COVID-19: prevalence, prognostic impact, pathophysiology, and management. Eur J Endocrinol. 185(4):R103-R111. doi: 10.1530/EJE-21-0281. 
14. Martino M, Falcioni P, Giancola G, Ciarloni A, Salvio G, Silvetti F, et al (2021). Sodium alterations impair the prognosis of hospitalized patients with COVID-19 pneumonia. Endocr Connect. 10(10):1344-1351. doi: 10.1530/EC-21-0411.

15. Fernandez Martinez A, Barajas Galindo D, Ruiz Sanchez J (2021). Management of hyponatraemia and hypernatraemia during the Covid-19 pandemic: a consensus statement of the Spanish Society for Endocrinology (Acqua Neuroendocrinology Group). Rev Endocr Metab Disord. 22(2):317-324. doi: 10.1007/s11154-021-09627-3.

16. Nair V, Niederman MS, Masani N, Fishbane S (2007). Hyponatremia in community-acquired pneumonia. Am J Nephrol. 27(2):184-90. doi: 10.1159/000100866.

17. Schuetz P, Haubitz S, Christ-Crain M, Albrich WC, Zimmerli W, et al (2013). Hyponatremia and antidiuretic hormone in Legionnaires' disease. BMC Infect Dis. 13:585. doi: 10.1186/1471-2334-13-585.

18. Krüger S, Ewig S, Giersdorf S, Hartmann O, Frechen D, Rohde G, et al (2014). Dysnatremia, vasopressin, atrial natriuretic peptide and mortality in patients with community-acquired pneumonia: results from the german competence network CAPNETZ. Respir Med. 108(11):1696-705. doi: 10.1016/j.rmed.2014.09.014.

19. Corrado RE, Lee D, Lucero DE, Varma JK, Vora NM (2017). Burden of Adult Community-acquired, Health-care-Associated, Hospital-Acquired, and Ventilator-Associated Pneumonia: New York City, 2010 to 2014. Chest. 152(5):930-942. doi: 10.1016/j.chest.2017.04.162.

20. Cuesta M, Slattery D, Goulden EL, Gupta S, Tatro E, Sherlock M, et al (2019). Hyponatraemia in patients with community-acquired pneumonia; prevalence and aetiology, and natural history of SIAD. Clin Endocrinol (Oxf). 90(5):744-752. doi: 10.1111/cen.13937.

21. Li Q, Wang Y, Mao Z, Kang H, Zhou F (2021). Serum Sodium Levels Predict Mortality in Elderly Acute Kidney Injury Patients: A Retrospective Observational Study. Int J Gen Med. 14:603-612. doi: 10.2147/IJGM.S294644.

22. Turgutalp K, Özhan O, Gök Oğuz E, Yılmaz A, Horoz M, Helvacı I, et al (2012). Community-acquired hypernatremia in elderly and very elderly patients admitted to the hospital: clinical characteristics and outcomes. Med Sci Monit. 18(12):CR729-34. doi: 10.12659/msm.883600.

23. Darmon M, Timsit JF, Francais A, Nguile-Makao M, Adrie C, Cohen Y, et al (2010). Association between hypernatraemia acquired in the ICU and mortality: a cohort study. Nephrol Dial Transplant. 25(8):2510-5. doi: 10.1093/ndt/gfq067.

24. Howanitz JH, Howanitz PJ (2007). Evaluation of serum and whole blood sodium critical values. Am J Clin Pathol. 127(1):56-9. doi: 10.1309/Q3Y27QQLEL19340A.

25. Wright JA, Cavanaugh KL (2010). Dietary sodium in chronic kidney disease: a comprehensive approach. Semin Dial. 23(4):415-21. doi: 10.1111/j.1525-139X.2010.00752.x.

26. Krekels MM, Kroon AA, de Leeuw PW (2015). Sodium Intake as a Modulator of Kidney Function. Curr Hypertens Rev. 11(1):57-60. doi: 10.2174/1573402111666150530204512.

27. Almirall J (2016). Sodium Excretion, Cardiovascular Disease, and Chronic Kidney Disease. JAMA. 316(10):1112. doi: 10.1001/jama.2016.11553. 
28. Richardson S, Hirsch JS, Narasimhan M, Crawford JM, McGinn T, Davidson KW, et al (2020). Presenting Characteristics, Comorbidities, and Outcomes Among 5700 Patients Hospitalized With COVID-19 in the New York City Area. JAMA. 323(20):2052-2059. doi: 10.1001/jama.2020.6775. Erratum in: JAMA. 2020;323(20):2098.

29. Zimmer MA, Zink AK, Weißer CW, Vogt U, Michelsen A, Priebe HJ, et al (2020). Hypernatremia-A Manifestation of COVID-19: A Case Series. A A Pract. 14(9):e01295. doi: 10.1213/XAA.0000000000001295.

\section{Figures}

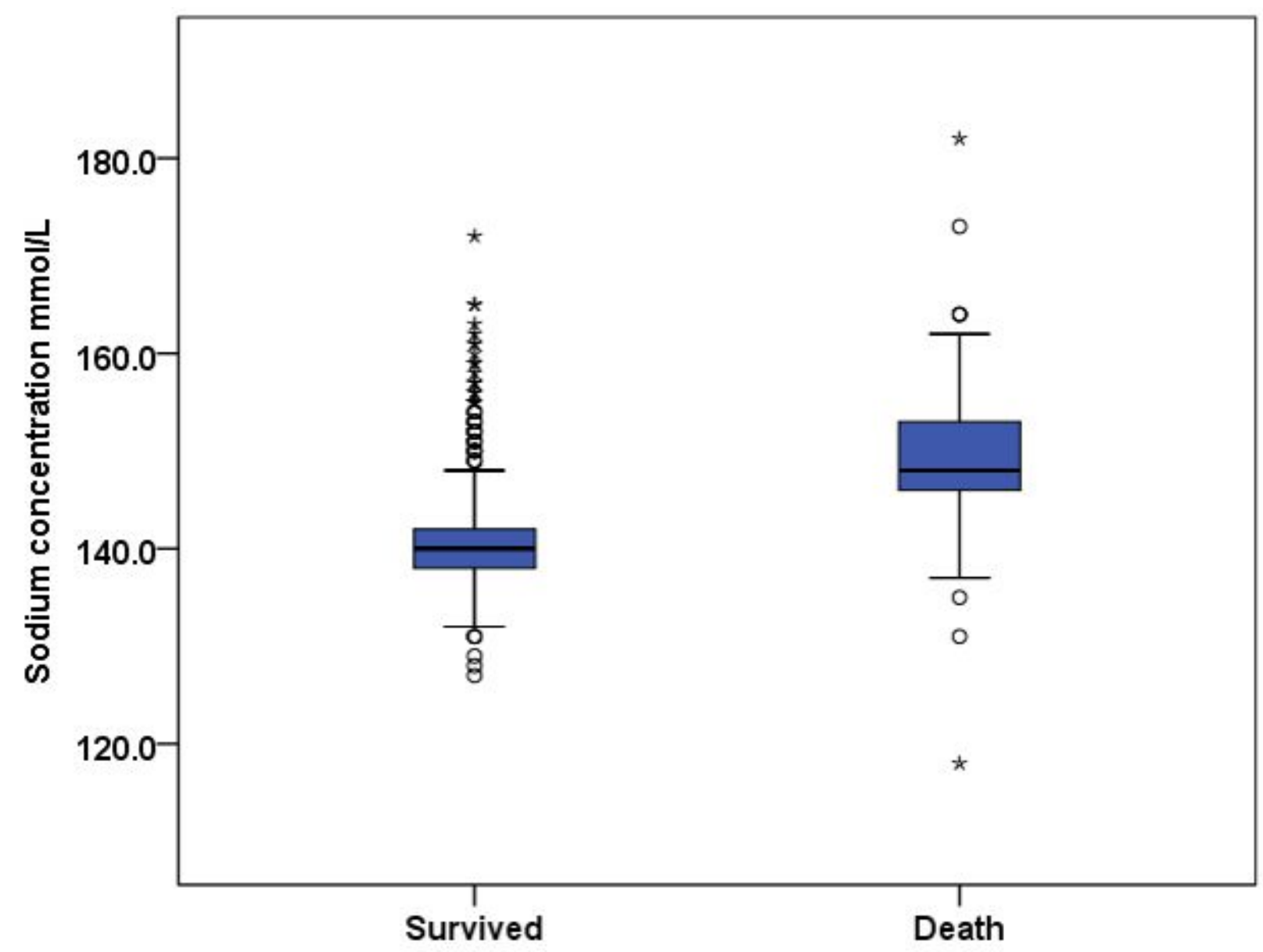

Figure 1

Comparison between sodium concentrations in survived and death in patients with COVID-19. 


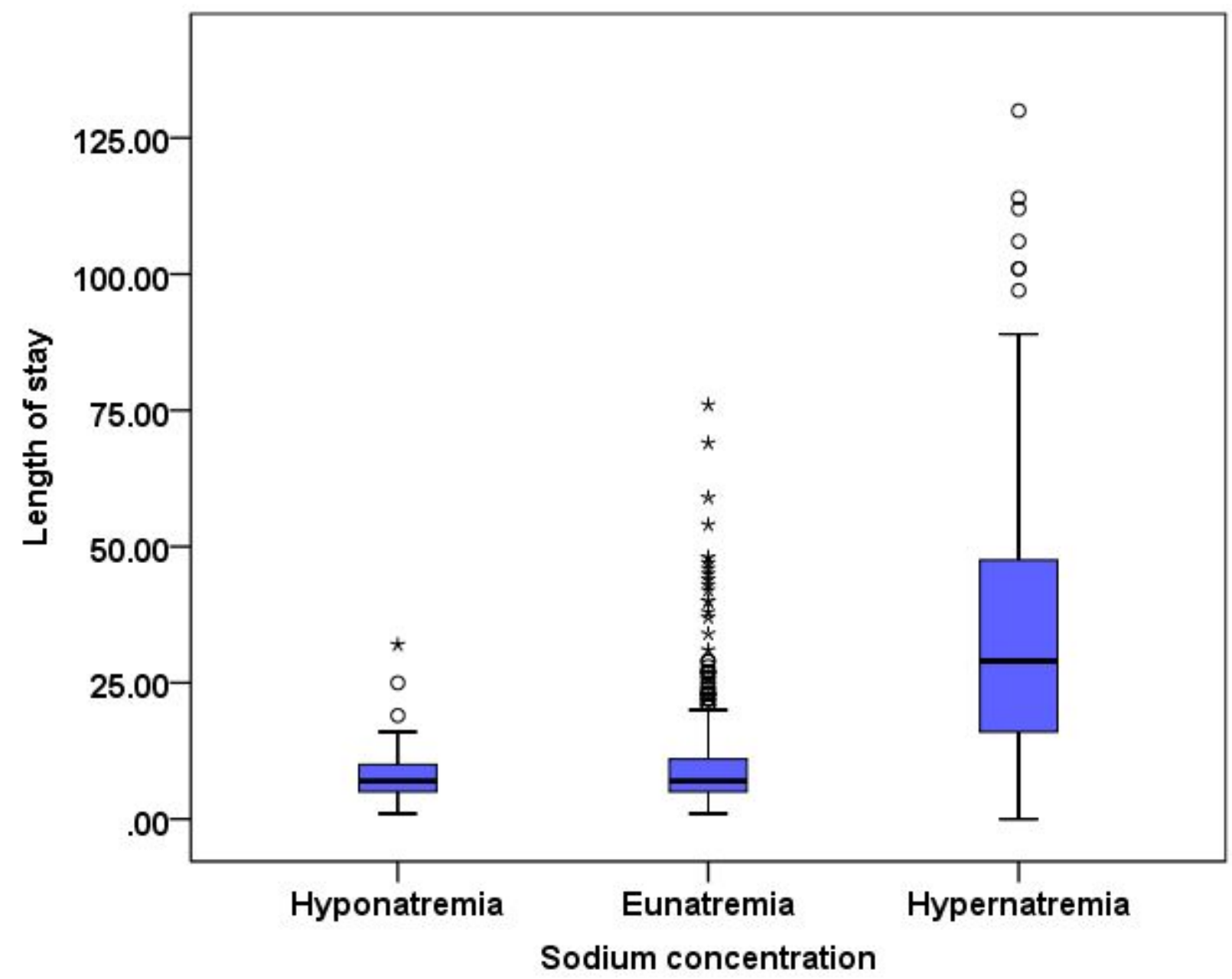

Figure 2

Comparison between sodium concentrations and length of stay in patients with COVID-19. 


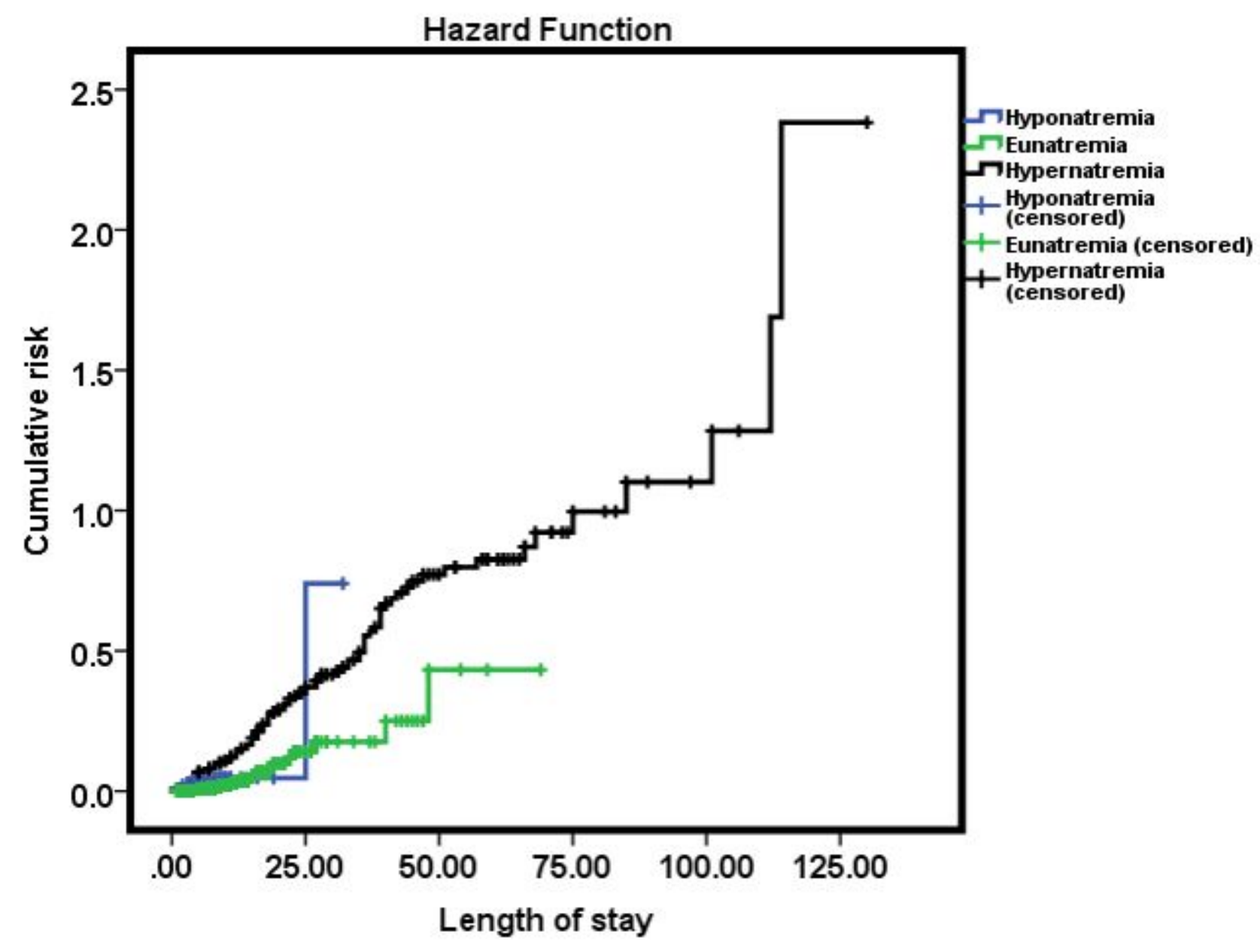

Figure 3

Kaplan-Meier curve showing probability of death risk based on serum sodium status in patients with COVID-19.

\section{Supplementary Files}

This is a list of supplementary files associated with this preprint. Click to download.

- TableS1.docx

- TableS2.docx

- TableS3.docx

- Tables4.docx 